\title{
Experiencias y propuestas para nuevos modelos de producción, difusión y comercialización de la Edición Contemporánea de Arte. Experiences and proposals for new models of production, diffusion and commercialization of the Contemporary Art Edition.
}

\author{
Antonio Damián Ruiz \\ Escuela de Artes José Nogué, Jaén, España \\ redlibrodeartista@gmail.com
}

Recibido 29/052017

Revisado 05/06/2017

Aceptado 07/06/2017

Publicado 15/06/2017

\section{Resumen}

Este artículo está diseñado para transferir la información necesaria que permita la transformación de la redlibrodeartista.info en una herramienta eficiente que pueda ser gestionada de manera colaborativa. Es un documento abierto, sujeto a las aportaciones de terceros, tanto en la evaluación de las actividades realizadas, como en el diseño y planificación de los flujos de trabajo futuros.

Parte del supuesto que considera a la
Abstract

This article is designed to transfer the required information that allows the transformation of redlibrodeartista.info in an efficient tool that can be managed in a collaborative way. It's an open document, subject to third party contributions, in the evaluation of conducted activities as well as the design and planning of workflows.

Part of the assumption which considers contemporary art edition a cultural industry, suggests analyzing it as any productive sector in which many specialists with high qualification

Para citar este artículo

Damián Ruiz, A. (2017). Experiencias y propuestas para nuevos modelos de producción, difusión y comercialización de la Edición Contemporánea de Arte. Tercio Creciente, 13, págs. 7-30. https://dx.doi.org/10.17561/rtc.mextra1.1 
edición de arte contemporánea como como una industria cultural, propone analizarla como a cualquier sector productivo en el que intervienen múltiples especialistas con una alta cualificación y que puede, si implementa unas estrategias adecuadas de formación, producción, difusión y comercialización, revitalizar un mercado, a través de los recursos que nos ofrecen las tecnologías digitales. take action and which may, if the right educational, production, propagation and marketing strategies are applied, revive a market, through the resources that digital technologies can offer.

Palabras clave / Keywords

Edición de arte, producción, difusión, comercialización, redlibrodeartista.org.

Contemporary art edition, production, diffusion, commercialization, redlibrodeartista.org.

Damián Ruiz, A. (2017). Experiencias y propuestas para nuevos modelos de producción, difusión y comercialización de la Edición Contemporánea de Arte. Tercio Creciente, 13, págs. 7-30. https://dx.doi. org/10.17561/rtc.mextra1.1 


\section{1.- La redlibrodeartista, un espacio de trabajo para comunes digitales.}

"Las condiciones materiales en las que se produce, distribuye y accede a la cultura se han visto modificadas drásticamente en las últimas dos décadas. Las tecnologías digitales y su articulación en red han sido las principales responsables de esto. Puede hablarse, así, de una reconfigurada ontología de la cultura debida a la digitalización, cuyo rasgo más evidente es la desobjetualización. Esta condición de las producciones digitales abre un abanico de posibilidades para la transformación de las prácticas culturales y plantea un serio reto para determinar y controlar su propiedad.

\footnotetext{
"Es precisamente en torno a ella que se conforman nuevas formas de asociación, tramadas en torno a los comunes digitales y otros modos de actuación colectivos como las asambleas o las redes difusas”. (López Cuenca, 2016:5)
}

Este texto de Alberto López Cuenca extraído del libro: Los comunes digitales: Nuevas ecologías del trabajo artístico, nos sitúa en el escenario en el que se han movido las actividades realizadas desde la redlibrodeartista en estos últimos diez años y nos da las claves para el diseño de las estrategias que propondremos para hacer de la edición de arte una industria cultural sostenible.

La crisis económica que hemos vivido, han cerrado muchos talleres y galerías, precarizando la actividad de los que han conseguido sobrevivir. Esto ha provocado la desestructuración de una actividad, que realmente no había conseguido nunca, ni en los mejores tiempos de boom económico, estar vertebrada como una industria cultural.

En relación proporcionalmente inversa al cierre de espacios expositivos y de talleres, se ha acudido a Internet, las redes sociales, lo "virtual", como remedio con el que aliviar la frustración de de no ver cumplidas las expectativas profesionales, o peor aún, que proyectos que había costado mucho tiempo y esfuerzo construir, se desmoronasen.

Toda esa frustración y la esperanza de que los medios digitales permitirían encontrar nuevos mercados fue la gasolina con la que funcionaron las actividades desarrolladas entre el 2009 y el 2014 en nuestra redlibrodeartista.

\section{2.- Antecedentes del proyecto.}

El sitio web, www.librodeartista.info, registrado como dominio el 21 de septiembre del 2006, fue el desencadenante de todas las actividades y flujos de comunicación que han tenido lugar después. Un lugar en el que se encontraron artistas, aficionados y curiosos con un denominador común; la ilusión, la expectativa de que había un espacio donde podrán ser reconocidos, exponer su obra e internacionalizarse sin salir de casa.

Esta ilusión difusa, de que "algo mejor de lo que estoy viviendo me va a pasar" hizo que muchos que no teníamos ninguna experiencia digital, ni colaborativa, y en muchos casos, 
ni siquiera relacionada con los libros de artista, se volcasen en proyectos comunes transnacionales.

Jim Lorena se incorpora en 2007 a la gestión del blog, realiza a mediados de 2008 una convocatoria "ellibroysuslecturaspuntoazul" relacionada con los libros de artista. Una convocatoria sin premio, sin promesas expositivas presenciales, tan sólo de exposición virtual que tuvo lugar el 29 de noviembre de 2008 y contó con una participación entusiasta de artistas que residían en EEUU, México, Colombia, Argentina, Chile, Italia y España. Lo que la hacía singular, era el hecho de haber sido realizada sin ningún tipo de ayuda por parte de instituciones, galerías o centros educativos. Fue un empeño personal de Jim que sólo cuando se percibió su trascendencia

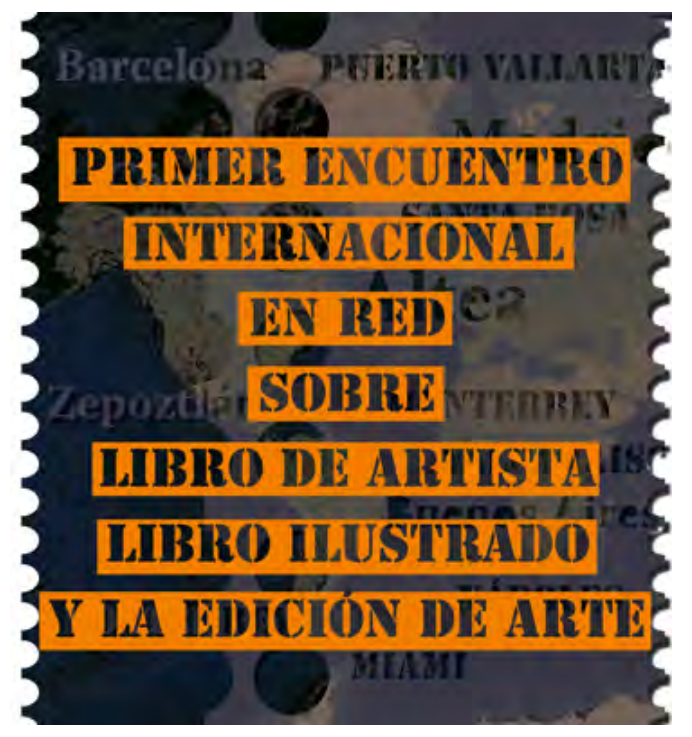

Cartel anunciador del Primer Encuentro interesó a una galería, la de Concha Pedrosa, y a una institución, la Facultad de Bellas Artes de Sevilla, que expusieron las obras seleccionadas.

Esta convocatoria fue un punto de inflexión en la dinámica relacional del colectivo que estaba interactuando a través de las redes. Se podía ir más allá de tener la sensación de que veían tu obra y de comentarios amables. Esas expectativas, podían verse concretadas en muestras reales, tanto virtuales como presenciales.

A comienzo del 2010 decidimos organizar un evento con espacios virtuales y presenciales, contando con el handicap de que las conexiones eran mucho más lentas que ahora, y los móviles y tabletas, tampoco tenían la capacidad actual. Además, tampoco existía ninguna otra experiencia previa similar a la que íbamos a desarrollar y que finalmente denominamos como el 'Primer Encuentro Internacional en Red sobre Libro de Artista, Libro Ilustrado y la Edición de Arte'.

El Primer Encuentro Internacional supuso el esfuerzo de los miembros de la red social alojada en el sitio http://librodeartista.ning. com - que ya contaba con más de un millar de miembros en octubre de 2009_, por difundir y profundizar en el conocimiento del género artístico de los libros de artista, los libros ilustrados y la edición de arte en general, haciendo visible el trabajo realizado en la red. Con este encuentro se intentó, al mismo tiempo, generar un mercado estable y sostenible de estos soportes creativos con el diseño de una serie de actividades realizadas en diversos ámbitos y diferentes ciudades, contando con unas líneas de trabajo y objetivos previamente estructurados y definidos. 
La coordinación fue de vital importancia para conseguir acometer estas actividades en grupo a través de las dinámicas que permiten la organización en red, el trabajo colectivo, la convergencia de medios y el copyleft. Nuestra forma de organización se correspondió a las nuevas estrategias que la red permite para la toma de decisiones conjuntas y el trabajo en colaboración.

Como grupo surgido en la red, ésta supuso el espacio de nuestras actividades y a través del cual intercambiamos experiencias y conocimientos, documentando aquellas que se hicieron de forma presencial. Asumimos que las ciudades son unidades culturales con mayor poder de interacción que los estados y, por lo tanto, los grupos de trabajo se crearon en torno a ellas. Desde Santa Rosa en la Pampa, hasta Miami, desde Ciudad de México hasta Nápoles pasando por Buenos Aires, Bogotá, Viña del Mar, Córdoba o Madrid. En todas ellas, los miembros de la red organizaron actividades en diferentes espacios dedicados a prácticas artísticas y docentes. Universidades, escuelas de arte, centros culturales, talleres de artes gráficas, librerías, galerías y asociaciones cedieron sus instalaciones para acoger distintas actividades presenciales, comprometiéndose a su digitalización y emisión a través de Internet.

\section{1. - La Vida Desatenta, La Vida Desatenta} ... Caminar y la Ley de Erradicación de la Vida Desatenta, un proyecto para desarrollar un trabajo continuado en red.

Esta actividad, que comenzó a raíz de una sugerencia de Antonio Navarro -en aquel momento profesor de la Facultad de Bellas Artes de Altea- ha sido la que mayor trascendencia y duración ha tenido. Pensada en un principio como convocatoria para realizar una exposición en la Escuela de Arte de Córdoba, con el tiempo se ha convertido en el proyecto vertebrador de la red., consolidando el espíritu de colaboración de esta red social, con miembros repartidos por diez países.

Fruto de este trabajo conjunto, se desencadenó un homenaje paralelo, independiente de cualquier encargo institucional, y con un diseño que permitió su realización sin subvenciones. Salvo la Escuela de Arte de Córdoba y la empresa Fotograbados Casares, que entendieron su espíritu y prestaron su apoyo y colaboración para hacer posibles las exposiciones y actividades necesitadas de una infraestructura y espacios físicos en la ciudad de Córdoba.

En concreto, se plantearon siete actividades anidadas y reunidas bajo el nombre de \#proyectomh, de las cuales sólo fructificaron cuatro:

- Una convocatoria por invitación directa realizada por José Emilio Antón para un libro ensamblado que tuvo por título "Hambre de Libertad". A ella fueron convocados artistas con una dilatada trayectoria dentro de las prácticas del libro de artista, el arte postal o la poesía visual como son César Reglero, Antonio Gómez, Clemente Padín, Felipe Ehrenberg, Pere Sousa y Jim Lorena.

- Una convocatoria abierta a los miembros de la red para el libro ensamblado En homenaje a Miguel Hernández. Este libro mantuvo el espíritu de las convocatorias heredadas del movimiento Fluxus, abierto a todo tipo de participación y sin selección 
previa. En él tuvieron cabida poemas visuales, arte postal, escritos o páginas de artista, entre otros formatos.

- $\quad$ Acción "No soy de un pueblo de bueyes" a cargo del poeta Antonio Gómez en la Escuela de Arte de Córdoba el 23 de abril, Día del Libro, en la que el autor recorrió los 481 metros que separan la Escuela de Arte Mateo Inurria de la Feria del Libro de Córdoba sobre una bovina de tejido no tejido (fliselina).

- La convocatoria para la exposición de libros de artista titulada La Vida Desatenta. Fueron invitados $50 \operatorname{artistas}^{1}$ de España, Italia, E.E.U.U., México, Colombia, Argentina, Chile y Brasil. Los criterios por los que se hicieron dichas invitaciones, tuvieron que ver con el deseo de juntar un grupo que viene desde diferentes prácticas artísticas, generaciones y países. Desde los pioneros en el trabajo en estos soportes como son; José Emilio Antón y Antonio Gómez (España); Felipe Ehrenberg (México) y Juan Carlos Romero (Argentina), a artistas emergentes que comenzaban sus trayectorias.

Simultáneamente a la exposición presencial, que se inauguró el 23 de abril en la Sala de Exposiciones de la Escuela de Arte Mateo Inurria, se presentó una virtual en el sitio web: www.lavidadesatenta.info en la que se recogía el proceso de creación de la obra y se intentaba responder a las preguntas: ¿quién? ¿cómo? ¿cuando? ¿dónde? se había realizado la obra.

Esta muestra que en principio fue pensada sólo como una actividad más, dentro del Primer Encuentro en Red, tuvo una ruta expositiva $^{2}$ que concluyó en la Sala Zabaleta de la Universidad de Jaén el 30 de enero de
2014, donde se unieron las dos primeras fases del proyecto y comenzó el tercero. En esta exposición se editó el catálogo en papel y se dio por concluida esta fase.

Una performance desencadena un proyecto itinerante de un liderazgo difuso, "La Vida Desatenta ... Caminar"

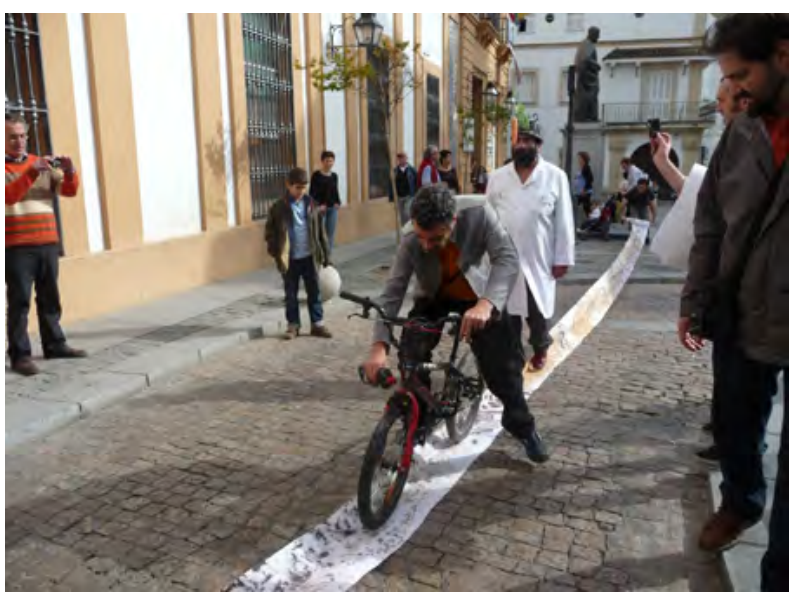

Performance de Antonio Gómez

La obra del poeta visual Antonio Gómez, una performance titulada: "No soy de un pueblo de bueyes", generó de forma natural, la segunda fase, denominada; "La Vida Desatenta ... Caminar”.

Antonio caminó desde la Escuela de Arte Mateo Inurria hasta el recinto de la Feria del Libro de Córdoba, sobre unas bobinas de tejido no tejido, previamente intervenidas con textos e imágenes realizadas con serigrafía. Durante el trayecto habría camino el artista Sancho Arnal, que había convertido una rueda 
www.terciocreciente.com de su bicicleta en una forma impresora con el texto : caminar por caminar. Durante la acción se realizó un video y al menos un millar de fotografías.

La dinámica de la acción estaba dividida en tres partes. En la primera, se desarrolló un trabajo colectivo realizado con alumnos de la escuela que intervinieron el soporte. En la segunda, Antonio Gómez, desde que pisa el tejido no tejido hasta que lo abandona, forma parte -durante los 30 minutos que dura la acción- de un libro de artista. El autor está dentro de la obra. En la tercera, se disemina esta obra en obras derivadas, los trozos del soporte que quedan a modo de reliquias y son reintervenidas por terceros, el video de la acción y los registros fotográficos que son reutilizados también para obras derivadas.

Las bobinas de tejido no tejido, resultantes de esta acción, 481 metros de largo por 45 centímetros de ancho, se enviaron a diferentes colectivos iberoamericanos, que las intervinieron y expusieron. Durante un tiempo, la ruta expositiva de la convocatoria (Córdoba, Granada, Jaén, Albacete, Hamburgo, Weimar) se solapó en el tiempo con la segunda fase del proyecto, concluyendo las dos en la exposición donde se hizo pública la convocatoria de la "Ley de Erradicación de la Vida Desatenta”.

Es esta fase, el principal objetivo fue conseguir que los participantes, tomasen el control del proyecto, se relacionasen y tomasen decisiones al margen de una coordinación unificada. Se enviaron fragmentos de las bobinas a Colombia, Argentina y España, con un listado de direcciones, para que fuesen reenviadas entre los participantes y pudieran ser intervenidas de forma colectiva. Se renunció a la monitorización de las rutas, con la expectativa de que se creasen por sí mismas, hecho que no ocurrió.

Posiblemente el problema de las aduanas y los costes de envío, tuvo algo que ver, pero en un análisis con mayor perspectiva, podemos decir que esos artistas o colectivos, no percibían su participación en la red como una manera de agruparse entre iguales, y no estaban predispuestos a la renuncia de la autoría.

Un recurso poético para desarrollar la tercera fase: "Ley de Erradicación de la Vida Desatenta”. Un proyecto de inteligencial creación colectiva

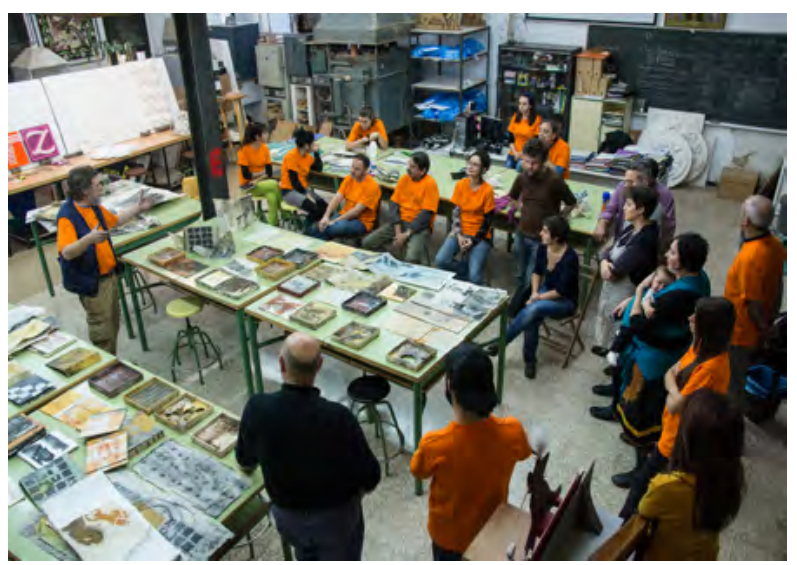

Reunión de trabajo en la Escuela de Arte José Nogué

Durante las Primeras Jornadas de Producción, Difusión y Comercialización de la Edición de Arte, realizadas del 29 de abril al 3 de mayo de 2013, se contempló la posibilidad 
de unir a los procedimientos de inteligencia/ creación colectiva, una estrategia de libre distribución de conocimiento. Durante estas jornadas, se diseñó un sistema de autoformación basado en los bancos comunes de conocimiento, diferentes expertos en materias nos reunimos, impartiendo talleres de nuestra especialidad y recibiéndolos de otros miembros del grupo.

La inteligencia/creación colectiva, generar y liberar conocimiento, crear un protocolo para coediciones realizadas por talleres en diferentes ciudades y países y la creación de una red de espacios expositivos fueron cuatro acciones asociadas que se plantearon como reto. Y la herramienta que se diseñó para su consecución fue la "Ley de Erradicación de la Vida Desatenta”.

El marco conceptual del proyecto consistió en la creación de una ley de iniciativa popular que permitiera a través de unas instrucciones prácticas y unas instrucciones técnicas, desarrollar una obra basada en los poemas de del libro "Caminar por Caminar" de Antonio Gómez y compartir los recursos técnicos de los procedimientos utilizados con el resto de los participantes en el proyecto.

Al ser el principal objetivo de esta actividad el desarrollo de un proyecto de inteligencia colectiva, se optó por no ejercer un liderazgo invasivo, algo que acabo no siendo del todo real, ya que en la sedes sí se ejerció. Esto, unido a que apenas hubo comunicación entre ellas, hizo que como proyecto de inteligencia/ creación colectiva, en cierto modo resultase fallido.

El análisis de esta experiencia nos indica que para conseguir este objetivo, debíamos realizar las siguientes modificaciones en el diseño del proyecto:

1. Debe cuestionarse el concepto de autoría, y trabajar tan sólo con aquellos artistas que estén dispuesto a hacerlo. No se debe forzar la participación de nadie que no esté dispuesto a renunciar a ella de forma puntual.

2. No es recomendable desarrollar proyectos simultáneos en diferentes ciudades y países. Sin embargo, los proyectos sí deben tener un componente híbrido de relación presencial y virtual.

3. El hecho de que sea un proceso de creación colectiva, no debe servir de excusa para no mantener un alto nivel de exigencia, tanto en los niveles de calidad de la ideación, como de la producción.

4. $\quad$ Se debe establecer un protocolo de trabajo para organización y difusión de la actividad con unos responsables de cada área y que permita una relación fluida entre grupos. Esto implica que los equipos estén bien coordinados entre sí y tengan estructuras similares.

\section{2.- Jornadas de Producción Difusión y} Comercialización de la Edición de Arte. Generar conocimiento, ensayo de la parte presencial de un posible título de experto universitario

La posibilidad de construir un mercado que permita la sostenibilidad de los proyectos relacionados con la edición de arte, pasa por el desarrollo de modelos de formación que se adapten a los contenidos multidisciplinares necesarios para gestionar la producción, 
difusión y comercialización como un todo. Que estos puedan modificar sus contenidos de forma permanente, adaptándose a los continuos cambios que se están produciendo y que tengan un componente virtual de formación continua y uno presencial de puesta en común.

Para estudiar un modelo de puesta en común, desarrollamos las I y II Jornadas sobre Producción, Difusión y Comercialización de la Edición de Arte, cuyo diseño se basó en los bancos comunes de conocimiento ${ }^{3}$, desarrollados a partir de las estrategias de la educación expandida 4 .

Maria del Mar Bernal, en su artículo "Los nuevos territorios de la gráfica: imagen, proceso y distribución”, describe de forma acertada, los motivos que dificultan la adaptación de los curriculum académicos a las necesidades reales de formación.

"Mientras que en el ámbito artístico estas inquietudes son bienvenidas, en el ámbito académico se produce cierta incomodidad ante el panorama de tener que actualizar toda una complicada maquinaria administrativa, de infraestructuras y de actualización del profesorado. El propio significado de Academia, entendida como sociedad científica y artística de autoridad, exige que todo lo nuevo haya de referenciarse en un conjunto de teorías ya estabilizadas, por lo que cualquier discurso ideológico se toma el doble de tiempo para asentarse.” (Bernal-Pérez, 2016: 86)

Ver el catálogo de talleres impartidos en el anexo I.

\section{3. Feria Cinco Sedes}

Fue un proyecto tan ambicioso y complejo que todavía se encuentra en fase de evaluación por sus participantes por lo que en este artículo solo se hará mención a cómo se definió, quién lo organizó y dónde.

\section{Definición:}

Una feria sobre los libros de artista realizados con los procedimientos de la Edición de Arte, que se realiza de forma simultánea en 9 espacios presenciales agrupados en 5 sedes que se relacionan entre ellas y con los visitantes, además del contacto físico, por medio de las redes sociales y páginas WEB.

Organizadores:

- Librodeartista.info (Antonio Damián, Jim Lorena y José Gallardo)

- Coordinación general: Antonio Damián.

- Comunicación: José Gallardo.

- Coordinación de contenidos: Jim Lorena.

Sede Jaén

- Asociación de Amigas y Amigos del Museo de Jaén.

- Escuela de Arte José Nogué

- Universidad de Jaén.

Sedes de México

Toluca

- Eje Gráfico (Alejandro Villalbazo, Carmen Razo y Armando Gómez)

- Taller La Pintadera

Guadalajara 
- Mónica Cárdenas (LIA)

\section{Michoacan}

- Ioulia Akhmadeeva (Facultad Popular de Bellas Artes UMSNH)

\section{Sede Pereira (Colombia)}

- Carlos Augusto Buriticá (Grupo de Investigación 700REPART de la Escuela de Artes Visuales de la Universidad Tecnológica de Pereira)

Sede Buenos Aires

- Graciela Marotta (Directora del Postgrado de Lenguajes Combinados de la IUNA)

Sede Lauderhill (Florida, USA)

- Liliana Gerardi (Lauderhill Center of Arts)

- Judith Gashghaine. Gestora cultural

¿Qué objetivos tuvo?

- Realizar una tomas de datos para su posterior análisis del panorama de la producción de libros de artista mediante los procedimientos de la edición de arte en Iberoamérica.

- Realizar una catalogación de los proyectos mas interesantes relacionados con este género. Respecto a:

- La formación reglada.

- Formación no reglada.

- Ferias y eventos de difusión.

- Talleres de gráfica adaptados a la producción en este soporte.

- Imprentas que trabajen en edición bajo demanda.

- Encuadernadores, maestros papeleros, etc, con procedimientos adaptados

- Diseñadores y fotógrafos con experiencia en el tema.

- Análisis y catalogación de espacios web existentes, relacionados con los libros de artista y la edición de arte

- Diseñar propuestas adaptadas a las tecnologías de la comunicación para crear estrategias de venta que combinen lo presencial y lo virtual.

- Fomentar el coleccionismo privado y público tanto en las sedes presenciales como a través de Internet.

- Fomentar las enseñanzas regladas o no que versan sobre estas prácticas

\section{3.- Profesionalizar la ilusión. La transformación de la red en una herramienta eficiente.}

Durante el 2016, los creadores de la redlibrodeartista, tomamos conciencia de que se había convertido en una red difusa y poco atractiva. Era el momento de tomar la decisión de enterrarla con honores, es decir, hacer una última actividad y a partir de ella realizar una publicación con la que catalogar y evaluar su eficacia. O bien transformarla en un nuevo modelo, que fuese eficiente, con metas y objetivos concretos, y que a partir de 
la experiencia previa, no fuesen excesivamente difíciles de cumplir.

Para seguir el trabajo, realizado de forma altruista y en nuestro tiempo libre, necesitábamos que el equipo de coordinación formado por Jim Lorena y Antonio Damián, se viera reforzado por otros miembros, que aportaran nuevas ideas y recursos. La persona con quien habíamos tenido mayor relación y ayuda, y que respondió a un correo destinado a la red, explicando la situación fue Antonio Alcaraz.

Decidimos, juntos, realizar una actividad que nos permitiese diseñar la nueva red, y comenzamos planificando el homenaje a nuestro común amigo el artista y tipógrafo alemán Emilio Sdun. De aquí surgió el Memorial Emilio Sdun y el número cero de la colección de publicaciones ensambladas "La Vida Desatenta" que lleva el nombre Bajo Presión. Ambas actividades relacionadas y que sirvieron para llenar de contenido la redlibrodeartista.

\section{1.- El Memorial Emilio Sdun. Origen de un} proyecto de museografía digital

Librodeartista.info Ediciones y el Departamento de Dibujo de la Facultad de Bellas Artes. Universitat Politècnica de València organizaron el Memorial Emilio Sdun, concebido como un conjunto de actividades cuyo objetivo fue recordar y homenajear la figura del artista y tipógrafo Dieter Emil Sdun, fallecido el 20 de enero de 2015, así como promover la creación y edición de libros de artista realizados con los procedimientos de la edición de arte contemporánea.

Para ello, se realizó una convocatoria que tomó como referencia el centenario de la inauguración del Cabaret Voltaire y el nacimiento del Dadaísmo y que fue expuesta en el Museo de Jaén de forma presencial, desde noviembre de 2016 a enero de 2017 y en www. redlibrodeartista.org de forma virtual. Esta muestra ha sido expuesta posteriormente en la Universitat Politècnica de València y ha estado acompañada por una selección internacional de libros tipográficos y por una retrospectiva de la obra de Emilio Sdun.

El objetivo de la convocatoria, ademas de rendir homenaje a Emilio Sdun, en el primer aniversario de su muerte y conmemorar el centenario del movimiento DADA pasa por:

- Estimular la creación de libros de artista hechos con los procedimientos de la edición de arte entre artistas, estudiantes de arte y sus profesores y profesoras.

- Que estas enseñanzas se afiancen en los centros de formación artísticos.

- Que los talleres de gráfica, diseñadores y fotógrafos aprendan prácticas de coedición.

- Que se establezca una relación fluida entre los centros educativos y los talleres a nivel internacional para diseñar estrategias formativas y de difusión, de forma que estos artistas y colectivos se conozcan entre ellos y comiencen a organizar proyectos colaborativos de producción, difusión y comercialización que permitan consolidar un mercado sostenible, estimulando el coleccionismo, tanto público como privado.

Estos objetivos prácticos, se canalizan a través de la creación de un espacio de museografía digital, que transforma esa red difusa que se aloja en http://librodeartista.ning. com en una más estructurada y eficiente, en el sitio www.redlibrodearitsta.org, un repositorio digital, en el cual se muestre de forma razonada un panorama de la creación de libros de artista 
realizados con los procedimientos de la edición de arte. Coordinado por un comité de expertos. Cuya composición responda a la forma asimétrica que ha conformado la redlibrodeartista desde su origen. En ella se habrían de integrar artistas, editores, docentes, impresores y estampadores, un reflejo de todo el ecosistema que conforma el mundo de la edición de arte cuando produce libros de artista.

Como apunta María del Mar Bernal en el artículo de su blog http://tecnicasdegrabado. es/2015/la-difusion-del-libro-de-artista-eninternet .

"La reputación en la red requiere tiempo y responde a un esfuerzo y capacidad considerables para filtrar información, enlazar a otras fuentes e, incluso, crear estados de reflexión.”

Este trabajo, o al menos parte de él, ya está realizado como hemos visto anteriormente, siendo prioritario consolidarlo, dotándolo de una utilidad práctica. Es decir, que sirva para que los artistas, los talleres y editores que se dedican a esta producción en el área que hemos acotado, procesual, conceptual y geográficamente, puedan consolidar sus proyectos y hacerlo sostenibles. Y que los coleccionistas, tanto públicos como privados, puedan tener una información adecuada a sus intereses.

\section{¿Qué equipo debe abordar este trabajo?}

En el artículo anteriormente citado de María del Mar Bernal, apunta:

"Para afrontar esta situación la actitud crítica y la solvencia de la institución o autor encargado de la difusión son fundamentales.
Se trata de depurar ese exceso informativo gestionando el equilibrio entre lo lúdico y lo profesional, entre lo decorativo y lo artístico, entre la creación y la producción.”

Y que no tenga una versión sesgada o gregaria de la producción. Un proyecto de museografía digital como el que se propone, debe gestionarse por un grupo, que responda a la asimetría de los autores y obras que van a ser expuestas. Este valor de la asimetría, como ya hemos visto antes, ha sido una de las fortalezas que ha tenido la red desde su comienzo. Desde departamentos de universidades a pequeños talleres que comenzaban su producción con pocos medios, todos eran comunes digitales, al abordar los proyectos o convocatorias propuestas.

Y además este equipo de gestión, debe tener el prestigio y reputación en red suficiente, como para que despierte la confianza de los autores y colectivos, y todos ellos vean en este espacio digital, un espacio común de reflexión, conocimiento y difusión. No se debe pretender en ningún momento, ser el espacio que aglutina a todos los autores que trabajan con los libros de artista realizados con los procedimientos de la edición de arte, solo de aquellos que quieren implicarse en el proyecto.

En el equipo de gestión, deben estar incluidos miembros que pertenezcan a centros educativos, editores, talleres y artistas. Asesorados por expertos en diseño de web y de flujos de contenido, programadores, correctores de estilo, fotógrafos, community manager, todos aquellos técnicos, que convierten una idea utópica en una herramienta eficiente. 
Sin embargo, esta necesidad de recursos humanos, hace este espacio de museografía digital, necesite del soporte de alguna o algunas instituciones y empresas que le den el respaldo.

La experiencia de la red, nos muestra un camino híbrido de actividades presenciales y virtuales. Un espacio meramente digital, se convierte en un ente abstracto que no implica la interacción de los participantes en él, solo clasifica y no genera.

Si para su construcción se ha recurrido al Memorial Emilio Sdun, para su desarrollo, se han diseñado dos actividades que describimos más adelante; la semana de la edición de arte y la edición de una colección de publicaciones ensambladas, bajo el nombre "La Vida Desatenta”

\subsection{La colección de publicaciones} ensambladas "La vida Desatenta" un proyecto de inteligencia colectiva que va mas allá del hecho editorial.

Para mantener vivo el espíritu de la red y generar conocimiento que sirva para diseñar e implementar estrategias de producción, difusión y comercialización de la edición de arte, es necesaria una actividad que permita la realización de encuentros y exposiciones de forma periódica.

Con tal fin se ha creado, la colección de publicaciones ensambladas bajo el nombre " $\mathrm{La}$ Vida Desatenta” que mantiene y evoluciona el espíritu de las tres fases del work in progress del mismo nombre.

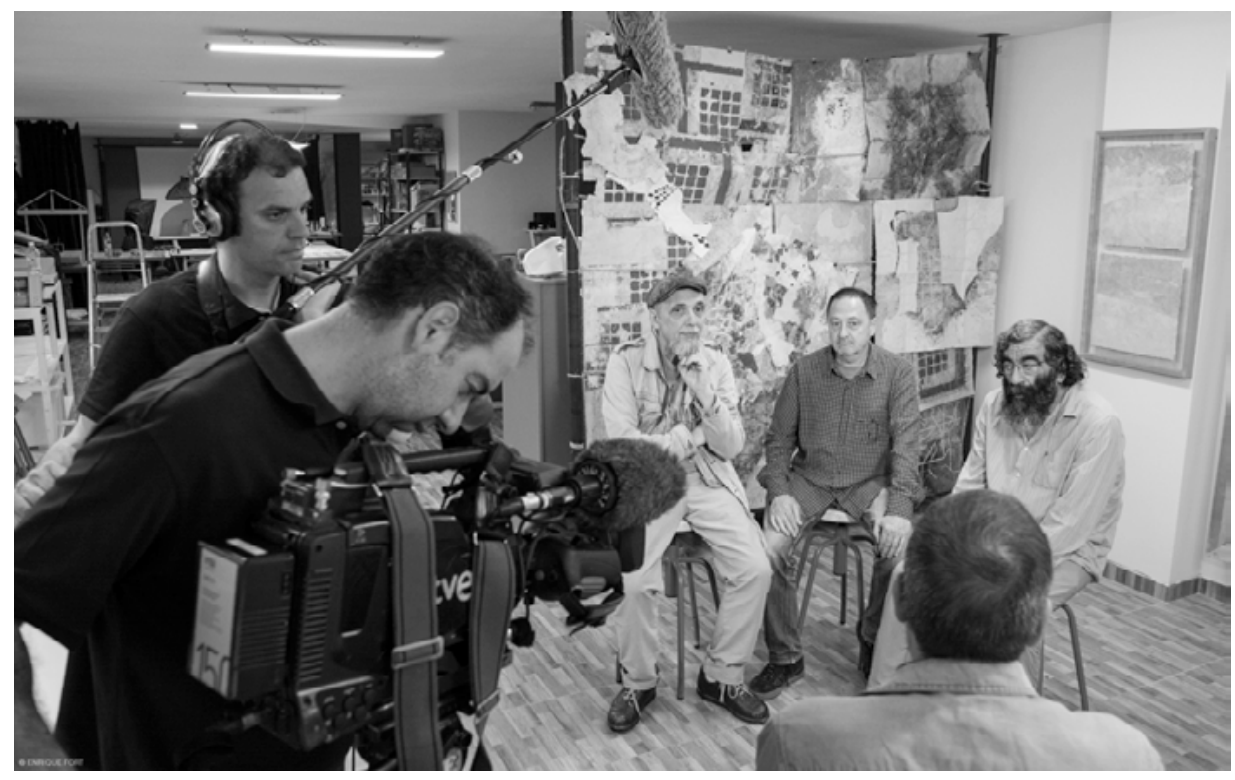

Pie de foto: Grabación del programa de TV2, la Aventura del Saber. 
Consiste en la publicación anual de la una colección de impresos, realizados sobre un tema, a partir de una serie de encuentros y jornadas, reunidos en una caja de $26 \times 26$ × 5 $\mathrm{cm}$. El comité editorial estará compuesto por Antonio Alcaraz, , Antonio Damián, Antonio Gómez y Antonio Terrada. Cada impreso podrá tener un formato libre siempre que pueda ser plegado y entre en la caja.

En este momento se está realizando la producción del número cero, de este proyecto, el cual, aunque mantiene el formato y la calidad de la obra no ha implementado aún el sistema de producción.

Este proceso de creación/inteligencia colectiva, se desencadenará en un encuentro, cuya fecha está aún por determinar, donde se establecerá el marco conceptual y la hoja de ruta de producción, en la que se incluye la serie de jornadas que se realizarán y los lugares en los que se llevarán a cabo. Manteniendo un flujo de comunicación permanente online y cuestionado el concepto de autoría.

Publicación ensamblada es un término que utilizamos para describir de forma posiblemente provisional, un procedimiento de trabajo, en el cual tras la elección de un tema por un comité editorial se procede a diseñar un contenedor que en el caso de la colección "La Vida Desatenta” será de $25 \times 25 \times 5$ centímetros, además de producción en el que, a partir de una serie de encuentros y jornadas, se realizarán los impresos realizado de forma colectiva y colaborativa.

El diseño de un proyecto editorial que tenga como afán, la generación de conocimiento en torno a la edición de arte, es completamente diferente a las que fijan su atención exclusivamente en el objeto artístico, en este caso el proceso es tan importante como el resultado.
Este proyecto es una de las herramientas, con las que se va a dinamizar el nuevo modelo de trabajo de la red, y para ello debe cumplir varios objetivos que normalmente no están asociados al quehacer editorial.

- Mantener un flujo de trabajo y reflexión, en torno a la edición de arte entre colectivos de forma expandida que sea visible al menos a los miembros de la red.

- Que en él participen otros artistas, además de los que tienen la edición de arte como práctica habitual, escritores, poetas y diseñadores.

- $\quad$ Diseñar una ruta de producción, en la cual, a partir del tema elegido se establezcan las sedes donde se realicen los encuentros o jornadas de producción, adaptando el modelo desarrollado en las 1a y 2a Jornadas de Producción, Difusión y comercialización de la Edición de Arte $^{5}$ realizadas en Jaén en 2013 y 2014.

- Asegurar la sostenibilidad

- Generar un marco de patrocinio que haga sostenible el proyecto dotando de una entidad jurídica visible que pueda asumir las ayudas

3.2.1. .- La Vida Desatenta, una colección editorial adaptado al flujo de trabajo de las publicaciones ensambladas.

Tras el análisis efectuado de los errores cometidos en el desarrollo del proyecto "Ley de Erradicación de la Vida Desatenta" vemos con claridad que los procesos de trabajo deben tener una capacidad organizativa clara y eficiente, diseñando un flujo de trabajo que sea lo suficientemente estructurado y flexible para 
organizar y gestionar proyectos complejos.

El primer paso en ese camino es la creación de un comité editorial multidisciplinar y con experiencia que proponga y dinamice las publicaciones. Tanto en el número 0 como en el número 1 se ha mantenido el siguiente organigrama.

- Coordinación general: asumida de forma conjunta para la colección, en cada número puede ser asumida por uno de los miembros o por un colaborador invitado.

- Comisariado o curaduría de la colección, Antonio Gómez.

- Coordinador de producción, Antonio Damián.

- Coordinador de la generación y registro de conocimiento, Antonio Alcaraz. Terrada.

- Coordinador de comunicación, Antonio

En cada número de la colección, se seguirá el siguiente protocolo:

1. Elección del tema para la publicación, elegida por el comité editorial o por una propuesta externa.

2. Designación de un coordinador de la edición , cuya misión es supervisar el desarrollo global del flujo de trabajo.

3. Nombramiento de un curador del ejemplar, que se encarga de la gestión del trabajo creativo:

- Selecciona los textos y elige a los artistas o colectivos que van a participar.

- Supervisa la calidad de la edición, en sus aspectos conceptuales y técnicos.

- Elabora los materiales de reflexión teórica sobre el proyecto.

4. Un coordinador de producción ${ }^{6}$ :

- Gestiona la temporización del proceso de producción.

- Organiza los encuentros presenciales de los participantes.

- $\quad$ Controla el reparto de materiales y trata con proveedores y patrocinadores. Un coordinador de comunicación ${ }^{7}$ :

- Gestiona la difusión del proyecto, antes, durante y después de su realización.

- $\quad$ Establece una comunicación eficiente y fluida entre el comité editorial y las sedes que participan y crea canales de comunicación entre las sedes.

- Coordina el diseño e impresión de las publicaciones digitales y en papel resultantes del proyecto.

5. Un gestor de generación y registro de conocimiento ${ }^{8}$.

- Realiza un diseño paralelo que convierte todo el conocimiento en publicaciones. Trabaja de forma independiente al resto del equipo, actuando como un evaluador externo.

\subsection{1.- Número 0. Bajo Presión. Ensayo general}

Todo proyecto necesita un ensayo, y una prueba de viabilidad. Especialmente en el caso que estamos describiendo en este artículo, que por su complejidad, parece difícilmente 
realizable. Por ello para el primer número de la colección, hemos suprimido gran parte del procedimiento de trabajo. Colaborando tan solo el colectivo formado en torno a la Universidad Politécnica de Valencia, los componentes de Jaén Edita y algunos profesionales invitados a colaborar y que centran su actividad en la impresión tipográfica con tipos móviles de imprenta (Familia Plómez, Industrias lentas, La seiscuatro).

Implementación del modelo diseñado para el sello editorial.

\section{Elección del tema:}

Esta publicación forma parte de las actividades del Memorial Emilio Sdun, hemos editado una publicación realizada con los mismos procedimientos técnicos que él uso en su labor artística como impresor y calígrafo. La composición tipográfica con tipos móviles, el fotopolímero y la serigrafía.

Coordinador de la edición:

Asumida por Antonio Alcaraz y Antonio Damián.

Curaduría del número:

De los textos:

Antonio Gómez realizó una selección de autores; Antonio Orihuela, Carlos Medrano, Elías Moro, Jordi Doce, José Ángel Cilleruelo, Luis Felipe comendador Sánchez, Montserrat Villar González, Tomás Sánchez Santiago, Uberto Stabile y él mismo.

De las imágenes:

Antonio Alcaraz y Antonio Damián seleccionaron los artistas visuales Enrique
Ferré, Andrea Familiar, La seiscuatro, Maribel Gomez Vilchez, Enrique Fort, Antonio Alcaraz, Vanesa Aguilar Hatero

Antonio Damián, Juanjo López, Famila Plómez, Jose Luis Armijo, Javier Jiménez, Agnieska Marcelak, Míriam del Saz, Viky Rodríguez, Elena Rodríguez, Industrias lentas, Alejandro Rodríguez León, Fernando Evangelio, Antonio Damián, Antonio Terrada y Jim Lorena.

\subsection{2.- Número 1. Ley de Erradicación de la} Vida Desatenta. Implementación del modelo de producción

Para implementar un modelo de producción tan complejo como el que se necesita para cumplir los objetivos propuestos en este número, resulta más práctico, retomar una experiencia que ya ha sido realizada y evaluada, y en la que la mayoría de los participantes colaboraron, por lo que ya tienen un entrenamiento en los protocolos de trabajo.

\section{Descripción.}

El número uno de la colección, funciona como un "remake" del work in progress que se desarrolló durante el 2014 con las mejores sugeridas en el análisis anteriormente expuesto y valorando que se trataba de un tema lo suficientemente bueno como para volver a intentarlo.

El texto en forma de Ley de Iniciativa Popular (L.I.P.) será rehecho, en esta ocasión por expertos juristas, que aportarán las correcciones que permitan elaborar un documento con validez legal.

Las instrucciones prácticas, es decir, la obra visual resultante de la lectura de los fragmentos de los poemas del libro "Caminar por Caminar" 
de Antonio Gómez, se mantendrán con algunas modificaciones, hasta completar un número entre 20 y 25.

Las instrucciones técnicas, es decir, la descripción de los procedimientos de trabajo, serán los artículos que elaborados por el equipo de gestión de conocimiento, pasarán a formar parte de la revista indexada Tercio Creciente y del repositorio de conocimiento que se creará en el sitio www.redlibrodeartista.org

La publicación se realizará en cinco jornadas, que seguirán el modelo desarrollado en las Jornadas de Producción, Difusión y Comercialización de la Edición de Arte, que se realizaron en Jaén en el 2013 y 2014. Teniendo tres sedes en España, una en México y otra en Colombia.

La publicación se realizará en cinco jornadas repartidas a lo largo de un año,en cada jornada, con una duración de cinco días, se dedicarán los dos primeros, a la puesta en común a través de las estrategias de los Bancos Comunes de Conocimiento, de recursos para la ideación, producción, difusión y comercialización, que van a ser aplicados en la producción de los impresos de la sede.

Por ejemplo, en la sede de Jaén, que inicia el proyecto, pueden realizarse actividades tan variadas, como talleres sobre la obra poética de Antonio Gómez, la interpretación artística de documentos legales en la historia del arte, procedimientos serigráficos aplicados a la edición de libros, realización de vídeo para la emisión de eventos en streaming, fórmulas de patrocinio para proyectos culturales, la edición de arte como industria cultural, etc

Los tres días siguientes, se dedicarán al diseño, creación de las matrices y edición de los impresos a los que se ha comprometido la sede.
Tiene especial importancia en la dinámica de trabajo en grupo distinguir la actividad del equipo de comunicación y el de gestión de contenido. En el primer caso, actúan retransmitiendo la actividad, como si se tratase de una televisión online. En el segundo, sería más bien una tarea documentalista, trabajando en paralelo documentos y transcribiendo las clases magistrales, talleres y entrevistas que se producen durante el evento, editando posteriormente audio, vídeos y documentos de texto que sirvan para su publicación en revistas indexadas y repositorios digitales. Estos dos equipos, deben tener responsables y componentes totalmente diferenciados.

\section{3.- Semana de la edición de arte contemporánea. La necesidad de lo presencial}

Todo el trabajo realizado de forma virtual, debe tener un evento o grupo de eventos que permitan el contacto personal de los participantes en el proyecto, para su autoformación y el diseño y temporización del trabajo a realizar.

Un encuentro de este tipo, debe tener una periodicidad anual, dividiéndose en dos fases; la primera de aspecto autoformativo, que siguiendo el modelo de los bancos de conocimiento, sirva para analizar el trabajo realizado, y fijar las estrategias y objetivos del siguiente curso. A esta primera fase la denominamos de forma provisional "Jornadas de Autoformación en la Producción, Difusión y Comercialización de la Edición de Arte” y debe contar con el respaldo de una institución educativa.

En la segunda parte de esta semana, se tendrá el contacto con el público, a través de un formato feria. Esta propuesta ha sido ya 
ensayada en el evento organizado en 2014 con el nombre Cinco Sedes.

\section{Una feria sin dogmas, simultánea e híbrida}

"Pero analizar la situación de la gráfica en estos eventos, abriría una interesante línea de investigación en un doble sentido: desde la estructura del mercado se identificaría quiénes son los principales agentes comercializadores de obra gráfica y el peso relativo de cada uno de ellos sobre la facturación global del sector. Es decir, se trataría de distinguir con claridad los roles y el protagonismo de proveedores (artistas), distribuidores (sector editoras, sector galerías y ferias, sector responsables de casas de subastas, sector instituciones) y clientes finales (coleccionistas). Resultaría también significativo conocer la realidad de la distribución de la gráfica en el mercado total para tomar conciencia de la necesidad de emprender acciones para promover la generación, difusión y conservación del patrimonio gráfico. Todavía parece que el sector debe ser estimulado por parte de muchos de los agentes implicados. Y como consecuencia de esto pudiera apuntarse en qué manera afecta -y si afecta- esta estructura a la configuración final de la obra gráfica.” (Bernal-Pérez, 2016: 83)

Cruzando las experiencias de Masquelibros y el experimento realizado en Cinco Sedes, podemos intuir que una feria que pretenda incidir de manera eficiente en las dinámicas del mercado, debe tener un componente virtual de latencia entre una fase presencial y la siguiente. Es difícil pensar, que un evento presencial dedicado a la edición de arte va a satisfacer las expectativas de los artistas y editores a nivel económico y de difusión, para los cuales en estos últimos años, no perder dinero y mantener la visibilidad, parece suficiente motivo para justificar una participación.

Los actuales canales de comunicación entre talleres y editores con sus clientes no se parecen a los que existían en los 90 en ferias como Estampa. En aquel momento, durante la feria, los participantes mostraban su nueva producción, que era vista por sus potenciales clientes por primera vez. También, era indispensable acudir, aunque solo fuese como espectador, para hacerse una idea de las tendencias y precios de las obras.

Hoy, en ferias como Art Libris o Masquelibros, las obras y los precios ya se han visto en las redes sociales o en las webs y la feria te proporciona el contacto personal con ellas y con los autores.

Pero toda esa dinámica de comunicación virtual y negocio online, no está estructurada ni ofrece el escenario de una producción que incentive el coleccionismo, sea público o privado.

Por ello, una feria que pretenda construir un mercado, y no ser un negocio en sí misma, debe prestar importancia a la actividad online durante todo el tiempo, mediante una estrategia de comunicación digital, que siga mostrando y comercializando la producción de los talleres y editores. Este objetivo, modifica radicalmente el planteamiento y desarrollo de la fase presencial, donde además del contacto con el público asistente, debe producir los materiales; registros fotográficos, vídeos, presentaciones, etc

Y en la medida de lo posible, también debe ser emitida en tiempo real por Internet, su área de influencia debe ir más allá de las personas que puedan acudir al evento presencial.

\section{¿Qué vende una feria de Edición de Arte?}

En principio podemos pensar que solo objetos artísticos, pero eso no es cierto. Quizá los editores, sean los únicos que cumplan este 
Estudio principio. La mayoría de los talleres, también venden formación no reglada y servicios de edición. Si acuden centros educativos, buscan matriculaciones en sus estudios. Y ya no digamos, los proveedores de materiales, cuyos clientes incluyen a los participantes del evento.

En resumen, se vende obra, servicios, formación y materiales, las cuatro fuentes de ingresos que hacen sostenible la economía de los talleres. Si una feria, se marca como objetivo, ser una herramienta eficiente y consolidar un marco productivo, no debe menospreciar ninguno de estos cuatro aspectos.

¿Cuáles serían los criterios de selección para los participantes en una feria de este tipo?

"La figura del comisario macla a toda una comunidad miscelánea bajo un parámetro contemporáneo y/o de vanguardia en el que artistas, galeristas y público, conforman un mosaico aparentemente bien ensamblado con mayor o menor tolerancia a la transgresión. Los agentes culturales seleccionan los artistas que deben adaptar su lenguaje a un espacio y un discurso determinado, lo que supone cierto riesgo de que se conviertan en meros ilustradores de la teoría del curador." (BernalPérez, 2016: 84)

María del Mar Bernal describe con exactitud el escenario que se produce en este tipo de eventos, donde es muy fina la línea que separa el criterio de selección en función de la calidad de las propuestas y una manipulación tendenciosa para resaltar unas tendencias sobre otras. Es cierto, que muchas veces, el criterio de selección está determinado por el afán de un curador por ilustrutar sus argumentos, pero también es cierto que un evento de este tipo, pierde toda su eficacia como dinamizador del mercado, si se convierte en un baratillo, en el cuál, se muestran todo tipo de obras, de forma masificada y sin ningún criterio de calidad.

\section{Conclusiones}

El mercado del arte en general, y la edición de arte como industria cultural, vive en un mundo que está en constante mutación, los circuitos a través de los cuales se comercializaba la producción, se ha transformado radicalmente. Los talleres, diversifican sus ingresos, ofreciendo además de la producción propia, servicio de formación y gestión cultural.

Esto implica, un nuevo modelo de talleres, constituidos por equipos multidisciplinares, en el que se integran especialistas en la gestión cultural, producción, difusión, y comercialización. Talleres que tienen la suficiente entidad y capacidad de trabajo, como para relacionarse con instituciones tanto educativas como culturales, en un nivel de igualdad. Este es el modelo de la Asociación Cultural Jaen Edita, diseñado como un proyecto sostenible y adaptado a las prácticas colaborativas.

Para que este tipo de talleres, puedan desarrollarse, es necesario consolidar una red en la que participen, además de los artistas, editores y talleres, la Universidad, las escuelas de arte, las empresas de artes gráficas y las instituciones.

Para consolidar este flujo de trabajo, iniciado en el 2009 y reforzado a partir del 2016, expongo las siguientes propuestas:

- Consolidar el grupo de trabajo realizado por miembros del Departamento de Dibujo de la Universitát Politécnica de València, del Máster en Investigación y Educación Estética: Artes, Música y Diseño de la Universidad de Jaén, de la Escuela de Arte José Nogué y de la Asociación Cultural Jaen Edita.

- A través del trabajo desarrollado en el 
número cero y en el número uno de la colección de publicaciones ensambladas la vida desatenta y en el memorial Emilio Sdun, integrar al grupo anterior, a componentes de otras universidades, escuelas de arte, talleres y editores, dotándolo de un carácter internacional, preferentemente iberoamericano.

- Darle una forma legal a ese grupo para la gestión del espacio de museografía digital: redlibrodeartista.org.

- Crear también a través de ese colectivo, materiales de investigación sobre formación, producción, difusión y comercialización de la edición de arte contemporánea, tanto para el monográfico en la revista de investigación en artes Tercio Creciente como para un repositorio de conocimiento localizado en el anterior sitio web.

- $\quad$ Establecer acuerdos de colaboración y patrocinio con empresas de Artes Gráficas que están liderando proyectos de I+D, que permitan, a través de un intercambio de conocimiento y servicios, obtener un mutuo beneficio.

- A partir de la colaboración anterior, desarrollar titulaciones de ámbito iberoamericano, de experto o especialista universitario en edición de arte.

- $\quad$ Crear un encuentro anual denominado "Semana de la Edición de Arte" que conste de una par-te del intercambio de conocimiento y una segunda de comercialización de productos y servicios.

\section{Notas}

1. Lista de autores en la web: www.lavidadesatenta.info

2. RUTA EXPOSITIVA DE LA MUESTRA

Escuela de Arte Mateo Inurria de Córdoba (Del 16 de abril al 8 de mayo de 2010)

Escuela de Arte de Granada (Del 13 de mayo al 7 de junio de 2010)

Universidad Popular de Albacete (Del 18 de octubre al 2 de noviembre de 2010)

Escuela de Arte de Jaén. Sala José Nogué (Del 8 al 24 de diciembre de 2010)

Biblioteca de la Universidad de Hamburgo (Del 8 de febrero al 18 de marzo de 2011)

Museo Provincial de Jaén (Del 24 de mayo al 20 de junio de 2011)

Biblioteca Municipal de Weimar, Alemania (Del 12 de noviembre al 3 de diciembre de 2011)

3. http://platoniq.net/es/bcc/p/2/que-es-banco-comun-de-conocimientos/

\section{4. http://www.zemos98.org/descargas/educacion_expandida-ZEMOS98.pdf}

5. En ellas durante 5 días, expertos en diferentes técnicas, siguiendo las estrategias de los bancos comunes de conocimiento, impartieron talleres o clases magistrales de su materia y recibiendolas de los otros participantes. 
6. De la experiencia resultante de la I y II Jornada sobre Producción, Difusión y Comercialización de la Edición de Arte, concluimos que deben separarse de forma clara el área de producción del evento presencial y la del evento en su fase virtual.

7.- El área de comunicación debe encargarse de crear y visualizar una imagen del proyecto que haga apetecible su participación, que durante su desarrollo todo el mundo se sienta informado de lo que está ocurriendo y orgulloso de ser partícipe, y que a su conclusión muestre con nitidez un relato coherente de la actividad desarrollada.

8.- El destino de este material será un repositorio digital aún por decidir su ubicación, así como el monográfico de la Revista de investigación Tercio Creciente de la Universidad de Jaén.

\section{Referencias}

Bernal-Pérez, Maria del Mar. Los nuevos territorios de la gráfica: imagen, proceso y distribución, (2016). Arte, Individuo y Sociedad, 28(1) 71-90,

López Cuenca, Alberto, (2016). “los comunes digitales: nuevas ecologías del trabajo artístico” . Centro de Cultura Digital, Secretaría de Cultura/Dirección General de Publicaciones.

Marín García, Teresa. Arte, creatividad y diseño.Arte, creatividad y diseño. Universidad Oberta de Catalunya. CC-BY-SA • PID_00165593

Baudrillard, J. (1993). La transparencia del mal (Ensayo sobre los fenómenos extremos). Barcelona: Anagrama.

Alcalá, J. R. (2006). “Explorando el laberinto. Gráfica, estampa y grabado en la sociedad digital”, en Grabado y Edición. Art Print Edition Magazine, pp. 6-16. 


\section{Anexo I}

I Jornadas sobre Producción, difusión y comercialización de la Edición de Arte (abril/mayo Talleres impartidos:

Adaptación de los talleres de gráfica a los nuevos soportes y materiales. Antonio Damián.

Maquetación adaptada a los libros de artista. Antonio Damián y Emilio Sdun.

Principios generales de caligrafía. Fabricación de herramientas. Emilio Sdun.

Realización de tipones, positivos y negativos a partir de originales caligráficos, para: Serigrafía, fotopolímeros y litografía en poliéster. Realización de pantallas serigráficas por método directo para caligrafía. Antonio Damián.

Las tintas, comparativas y nuevos materiales. Elección de las tintas para técnicas mixtas. (Antonio Damián)

Fabricación de papel: Papel con procedimientos orientales para fondinos. Papel blando de algodón para tipografía y gofrados. (Jim Lorena)

Creación de perfiles de color para papeles artesanales. (Manuel Torres)

El fotopolímero en el grabado en hueco y en relieve. (Paco Mora)

Fotopolímeros, creación de imágenes digitales con placas de flexografía. (José Gallardo y Antonio Damián)

Monotipos en gelatina. (Jim Lorena)

Caligrafía oriental. (Carlos Buriticá)

Tipografía creativa con tipos móviles en sacapruebas. (Familia Plómez)

Siligrafía. (Alejandro Villalbazo) 
II Jornadas sobre Producción, difusión y comercialización de la Edición de Arte (mayo 2014).

Talleres impartidos:

Papel con procedimientos orientales. Taller de fabricación de papel. Taller de prensado y almidonado de papel. Jim Lorena

Plegados y trenzados de papel. Darío Zeruto

Flujos de producción en la Edición de Arte. Ejemplo y modelo: Libro para el proyecto de la Ley de Erradicación de la Vida desatenta. Antonio Damián.

Técnicas de kirigami. Carmen Razo.

Estructuras experimentales de encuadernación. Taller de encuadernación experimental. Albertina Tafolla.

Técnicas de siligrafía. Alejandro Villalbazo

Técnicas de serigrafía manual aplicada a plegados, kirigami y encuadernación experimental. Antonio Damián.

Reproducción de familias tipográficas en poliuretano. Eduardo Cano

Registro fotográfico y en vídeo de libros de artista para catálogos y WEB con equipos de bajo coste. Jose Gallardo

El libro de artista como original para ediciones de bibliófilo. Javiera Pintocanales 
Revista de Estudios en Sociedad,

Artes y Gestión Cultural

www.terciocreciente.com

http://revistaselectronicas.ujaen.es/index.php/RTC
Monográfico Extraordinario I

Julio 2017

\section{Estudio}

ISSN: 2340-9096

DOI: 10.17561/rtc.mextra1.1 